\title{
Contribuição para o conhecimento dos Parandrini da Costa Rica e do Panamá (Coleoptera, Cerambycidae, Parandrinae)
}

\author{
Antonio Santos-Silva ${ }^{1} \&$ Humberto J. Lezama ${ }^{2}$
}

\begin{abstract}
${ }^{1}$ Museu de Zoologia, Universidade de São Paulo. Caixa Postal 42494, 04218-970 São Paulo-SP, Brasil. toncriss@uol.com.br
${ }^{2}$ Museo de Insectos, Escuela de Agronomía, Universidad de Costa Rica. Apartado postal 2060, San Pedro, Montes de Oca, San José, Costa Rica. oncideres@gmail.com
\end{abstract}

\begin{abstract}
Contribution towards the knowledge of Parandrini of Costa Rica and Panama (Coleoptera, Cerambycidae, Parandrinae). Only four species of Parandrini were known in Costa Rica and Panama: three in Parandra Latreille, 1802 (of which only two are recorded to Panama), and one in Birandra Santos-Silva, 2002. This work records Parandra (Parandra) solisi (SantosSilva, 2007) for the first time to Panama, and adds two new species: Parandra (Parandra) gilloglyi sp. nov. from Panama, and Birandra (Birandra) boucheri sp. nov. from Costa Rica and Panama. Keys to the species of Parandra (Parandra) which occur in Costa Rica and Panama, and to the species of Birandra (Birandra) known in Central America are added.
\end{abstract}

KEYWORDS. Birandra; Cerambycidae; Parandra; Parandrinae; taxonomy.

RESUMO. Contribuição para o conhecimento dos Parandrini da Costa Rica e do Panamá (Coleoptera, Cerambycidae, Parandrinae). Somente quatro espécies de Parandrini eram conhecidas da Costa Rica e do Panamá: três em Parandra Latreille, 1802 (das quais só duas estavam registradas para o Panamá) e uma em Birandra Santos-Silva, 2002. Este trabalho registra Parandra (Parandra) solisi (Santos-Silva, 2007) pela primeira vez para o Panamá e acrescenta duas espécies novas: Parandra (Parandra) gilloglyi sp. nov., procedente do Panamá, e Birandra (Birandra) boucheri sp. nov., proveniente da Costa Rica e Panamá. São fornecidas chaves para as espécies de Parandra (Parandra) que ocorrem na Costa Rica e Panamá e para as espécies de Birandra (Birandra) conhecidas na América Central.

PALAVRAS-CHAVE. Birandra; Cerambycidae; Parandra; Parandrinae; taxonomia.

Recentemente, foram encontrados dois espécimes de Birandra Santos-Silva, 2002 da coleção do Museo de Insectos, Escola de Agronomía, Universidad de Costa Rica, San Pedro, San José, Costa Rica (MICR), que correspondiam a uma nova espécie.

Durante o processo de estudo da nova espécie, recebemos para identificação, uma pequena coleção de Parandrini do Panamá, coletados, principalmente, por Alan R. Gillogly, Curador de Entomologia do Orma J. Smith Museum of Natural History, Caldwell, Idaho, Estados Unidos (OJSM). Quase todos os espécimes dessa coleção, foram coligidos em troncos mortos, durante busca por passalídeos, quando, ocasionalmente, eram encontrados adultos de parandrinae escavando galerias na madeira, ou espécimes recém eclodidos. Posteriormente, Aaoron Smith e Gary L. Parsons [Albert J. Cook Arthropod Research Collection, Michigan State University, East Lansing, Michigan, Estados Unidos (ARCO)] e James Wappes [American Coleoptera Museum, San Antonio, Texas, Estados Unidos (ACMT)] enviaram material adicional, também para identificação.

Atualmente, são registradas quatro espécies de Parandrini para a Costa Rica e três para o Panamá: Parandra (Tavandra) polita (Say, 1835); Parandra (Parandra) glabra (De Geer, 1774); Parandra (Parandra) solisi (Santos-Silva, 2007) (somente na Costa Rica) e Birandra (Yvesandra) angulicollis (Bates, 1879). O registro de Parandra (Tavandra) longicollis Thomson, 1861, desde a Argentina até os Estados Unidos (Monné 2006), é questionável e já foi refutada para esse último país por Chemsak (1996). Acreditamos que a espécie realmente envolvida, na área da América Central e do Norte, é $P$. (T.) polita, ou seja, o registro de $P$. (T.) longicollis para essa região, baseou-se em espécimes incorretamente identificados. Da mesma forma, o registro de Birandra (Birandra) punctata (White, 1853), que só aparece em Monné \& Giesbert (1994) e Monné $(2002,2006)$, embora não possa ser desconsiderado, permanece duvidoso.

Neste trabalho são descritas duas novas espécies, confirmada a ocorrência das quatro mencionadas acima, entre as quais, uma recentemente descrita da Costa Rica e registrada pela primeira vez para o Panamá. As referências bibliográficas restringem-se a descrição original e ao catálogo de Monné (2006). Somente os espécimes examinados foram plotados nos mapas.

Os acrônimos utilizados ao longo do texto, além dos cinco relacionados acima, correspondem as seguintes instituições: CASC, California Academy of Sciences, San Francisco, Califórnia, Estados Unidos; INBio, Instituto Nacional de Biodiversidade, Santo Domingo de Heredia, Costa Rica; MIUP, Museo de Invertebrados G. B. Fairchild de la Universidad de Panamá, Cidade do Panamá, Panamá, Panamá; MZSP, Museu de Zoologia, Universidade de São Paulo, São Paulo, São Paulo, Brasil; STRI, Smithsonian Tropical Research Institute, Ilha Barro Colorado, Panamá, Panamá; TAMU, Texas A\&M University, College Station, Texas, Estados Unidos; USNM, National Museum of Natural History, Washington, D. C., Estados Unidos. 


\section{Parandra (Parandra) gilloglyi sp. nov.} (Figs. 1, 2, 5-6, 10, 13)

Etimologia. Homenagem a Alan R. Gillogly, pelo empréstimo do material para estudo e doação de todos os tipos para diversas instituições.

Tegumento castanho-escuro; são enegrecidos: parte frontodorsal da cabeça, genas, mandíbulas, margens do pronoto, parte do escutelo, sutura elitral, margens dos episternos e das cavidades coxais, extremo apical dos fêmures, extremo basal das tíbias e partes dos tarsos.

Fêmea (Fig. 1). Face dorsal da cabeça com pontos finos e dispersos, gradualmente mais grossos e abundantes em direção às laterais; área entre os lobos oculares superiores, próxima à carena ocular e ao clípeo, com depressão circular a cada lado, moderadamente rasa; região central do labro projetada e com ápice estreitamente truncado; carena ocular saliente. Área atrás dos olhos com pontos grossos, abundantes, em parte confluentes. Olhos grandes; lobo ocular inferior não notavelmente mais largo do que o superior. Submento com pontos grossos, mais abundantes nas laterais e esparsos na região central em direção à gula; margem anterior não elevada (às vezes, suavemente elevada), lisa.

Mandíbulas (Fig. 5) com 0,8 vezes o comprimento da cabeça; pontuação abundante, fina na região dorsal, gradualmente mais grossa em direção às laterais; carena dorsal baixa, larga, restrita ao terço basal; margem interna sem reentrância acentuada na região central, com um dente moderadamente pequeno na região central e dois dentes na base; dente interno do ápice pequeno e voltado para frente.

Antenas curtas (não atingem o meio do protórax), estreitadas em direção ao ápice; antenômeros IV-V mais largos do que longos; área sensorial ventral dos antenômeros III-XI dividida por carena, não notavelmente visível em vista lateral; pilosidade esparsa e moderadamente curta na região dorsal e nas laterais, mais longa e abundante no ápice anteroventral dos antenômeros III-X.

Protórax transversal. Pronoto com pontuação muito fina e apenas abundante; margem anterior côncava; ângulos anteriores e posteriores arredondados; ângulos laterais marcados, obtusos; marginação lateral completa, voltada para cima da base aos ângulos laterais. Élitros com pontuação fina e moderadamente abundante. Metepisternos com pontos grossos, rasos, moderadamente abundantes junto às cavidades mesocoxais e gradualmente mais esparsos em direção às cavidades metacoxais; pilosidade presente apenas no quarto próximo às cavidades mesocoxais. Metasterno glabro, com pontuação muito fina e dispersa.

Macho (Fig. 2). Região atrás dos olhos com pontos grossos, profundos, confluentes e abundantes. Mandíbulas mais longas ou aproximadamente tão longas quanto à cabeça; margem interna nos machos major com um dente pequeno na região central, proporcionalmente maior e mais apical nos machos minor. Marginação lateral do pronoto completa (da base ao ápice). Antenas (Fig. 10) como nas fêmeas.

Dimensões em mm (macho/fêmea). Comprimento total (incluindo mandíbulas), 26,2-36,9/28,6-36,7; largura do protórax entre os ápices dos ângulos laterais, $7,3-10,2 / 8,2-10,3$; largura umeral, 6,9-9,7/8,3-10,5; comprimento elitral, 13,9-18,8/17,5-21,7.

Material-tipo. Holótipo fêmea, proveniente do PANAMÁ (Fig. 12), Chiriquí: Reserva La Fortuna, $8^{\circ} 43.195^{\prime} \mathrm{N}-82^{\circ} 14.247^{\prime} \mathrm{W}, 1225 \mathrm{~m}$, luz UV, 29.VIII.2008, Alan R. Gillogly col. (USNM). 26 Parátipos (9 machos, 17 fêmeas) - PANAMÁ (Fig. 12), Chiriquí: Reserva La Fortuna, $8^{\circ} 43.195^{\prime} \mathrm{N}-$ $82^{\circ} 14.247^{\prime} \mathrm{W}, 1225$ m, luz UV, macho, 29.VIII.2008, Alan R. Gillogly col. (USNM); idem, fêmea, 29.VIII.2008, Alan R. Gillogly col. (MIUP); idem, fêmea, 29.VIII.2008, Alan R. Gillogly col. (STRI); idem, 1 macho, 1 fềmea, 31.VIII.08, Alan R. Gillogly col. (MZSP); idem, fêmea, 31.VIII.2008, Alan R. Gillogly col. (CASC); idem, Estação Biológica, $08^{\circ} 43.18^{\prime} \mathrm{N}-82^{\circ} 14.17^{\prime} \mathrm{W}$, 1189 m, luz UV/MV, macho, 4-8.VIII.1999, Alan R. Gillogly col. (INBio); idem, fêmea, 4-8.VIII.1999, Alan R. Gillogly col. (TAMU); idem, 843.2’ N - 82 $2^{\circ}$ 14.247'W, 1225 m, 5 machos, 8 fêmeas, 29.VIII-02.IX.2008, S. M Smith col. (ARCO); idem, 1 macho, 1 fêmea, 29.VIII-02.IX.2008, S. M. Smith col. (MZSP); idem, fêmea, 29.VIII-02.IX.2008, S. M. Smith col. (MICR). Darién: Cana, "Field Station", $7^{\circ} 45.367^{\prime} \mathrm{N}-77^{\circ} 41.050^{`} \mathrm{~W}, 530 \mathrm{~m}$, fêmea, 2.V.2008, Alan R. Gillogly col. (MZSP); idem, atraído por luz, fêmea, 9.V.2008, Alan R. Gillogly col. (USNM).

Discussão. Parandra (Parandra) gilloglyi sp. nov. (Fig. 5) e P. (Tavandra) imitatrix (Santos-Silva, 2005), são as únicas espécies conhecidas no gênero, cujas fêmeas não apresentam reentrância acentuada na região mediana da margem interna das mandíbulas, o que as torna semelhantes àquelas das fêmeas de Birandra Santos-Silva, 2002. Esse caráter permite separar facilmente as fêmeas da nova espécie de todas as outras do subgênero [e.g.. P. (P.) glabra (Fig. 6)]. Os machos diferem quase exclusivamente de todas as espécies de Parandra (Parandra), pela forma das antenas (Fig. 10) que são visivelmente estreitadas em direção ao ápice e pelo formato do antenômero XI. Estes são os únicos caracteres confiáveis para separar os machos de $P$. (P.) gilloglyi sp. nov. das espécies que ocorrem na mesma região: P. (P.) glabra (De Geer, 1774) (Fig. 11) e P. (P.) solisi (Santos-Silva, 2007).

\section{Parandra (Parandra) solisi (Santos-Silva, 2007)} (Figs. 12-13)

Hesperandra (Zikandra) solisi Santos-Silva, 2007: 2, 8, 9, Figs. 1-9. Parandra (Parandra) solisi; Santos-Silva \& Shute, 2009: 31.

Descrita de áreas montanhosas, acima de $1000 \mathrm{~m}$ de altitude, da Costa Rica (Puntarenas e Cartago) e registrada pela primeira vez para o Panamá, também em zonas de altitude.

Material examinado. COSTA RICA (Fig. 12), Puntarenas: Reserva Biológica Monteverde, Estação La Casona, 1520 m, 2 fêmeas (holótipo e parátipo), XI.1990, Mauricio Ramirez col. (INBio); idem, parátipo fêmea, IX.1991, N. Obando col. (MZSP); idem, 3 parátipos fêmea, IX.1991, N. Obando col. (INBio); idem, parátipo fêmea, VIII.1992, N. Obando col. (INBio); idem, parátipo fêmea, VIII.1993, N. Obando col. (INBio); idem, 2 parátipos fêmea, 6-25.VI.1994, L. Martínez col. (INBio); idem, parátipo fêmea, 20.IX-8.X.1999, J. Rodríguez col. (INBio); idem, parátipo fêmea, 28.V-12.VI.1999, J. Rodríguez col. (INBio); San Luis, Reserva Biológica Monteverde, 1040 m, parátipo fêmea, VIII.1992, Z. Fuentes col. (INBio). Cartago: Parque Nacional Tapantí, Quebrada Salto, 1400 m, parátipo fêmea, 12.IX.1991, F. Muñoz col. (INBio); idem, Quebrada Segunda, parátipo fêmea, VII.1991, G. Mora col. (INBio); idem, 1250 m, parátipo fêmea, V.1992, G. Mora col. (INBio); idem, parátipo fêmea, 18-28.II.1993, F. A. Quesada col. (INBio); idem, 1150 m, parátipo fêmea, VIII.1994, G. Mora 

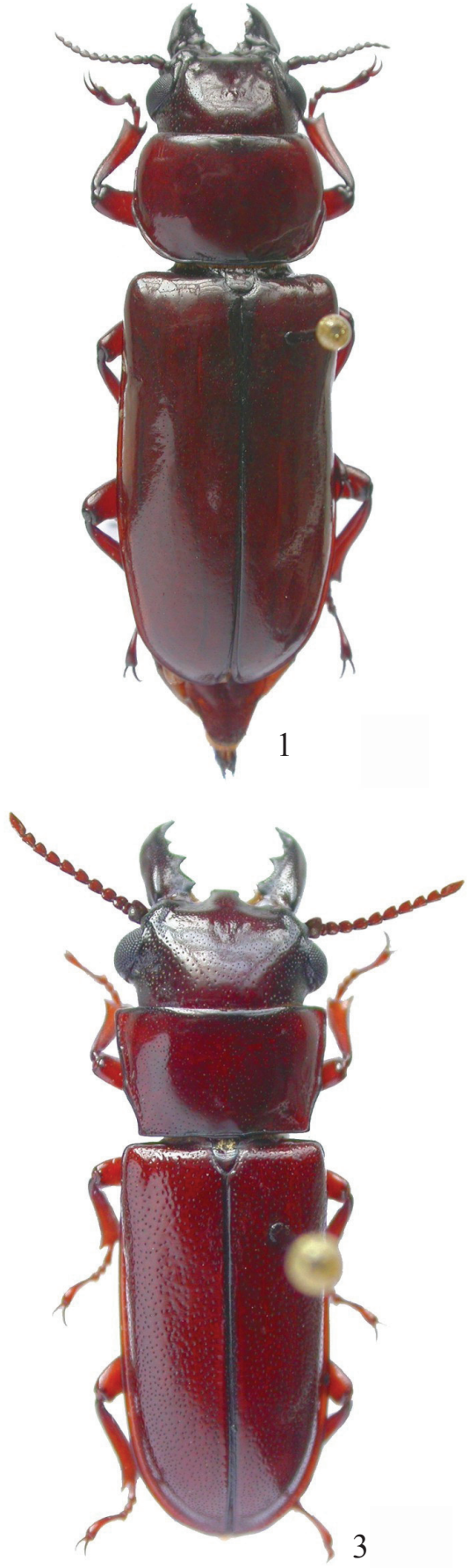
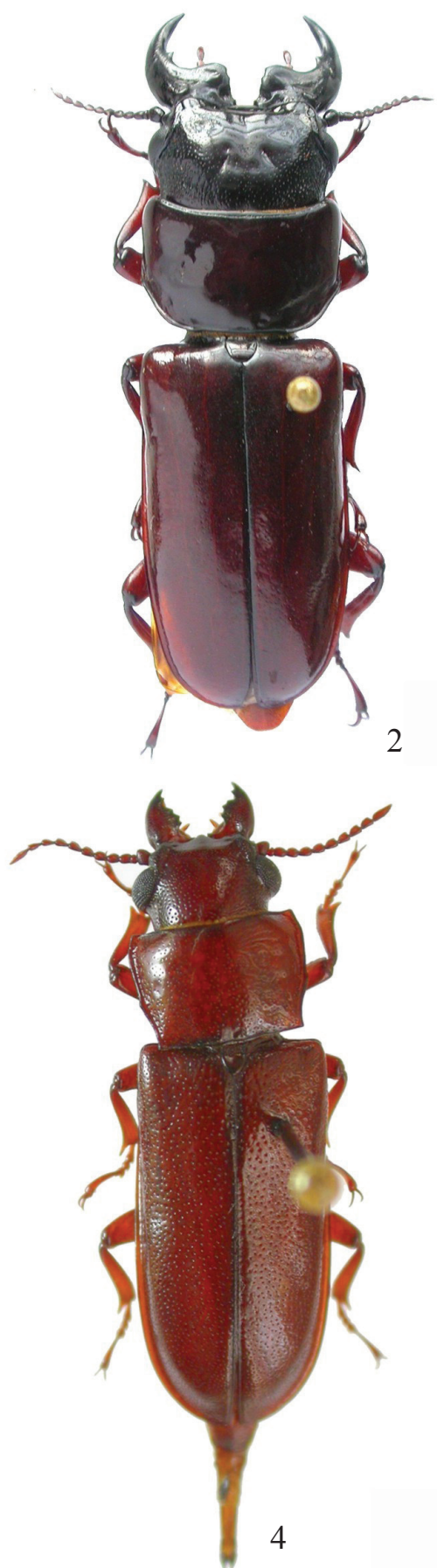

Figs. 1-4. 1, Parandra (Parandra) gilloglyi sp. nov., holótipo fêmea, comprimento 32,2 mm; 2, P. (P.) gilloglyi sp. nov., parátipo macho, comprimento 36,7 mm; 3, Birandra (Birandra) boucheri sp. nov., holótipo macho, comprimento $21,9 \mathrm{~mm} ; 4$, B. (B.) boucheri sp. nov., parátipo macho, comprimento 20,9 mm.

col. (INBio). São José: Miravalles, parátipo fêmea, 9.V.1931, A. Alfaro col. (MZSP); Parque Nacional Bráulio Carrilo, Estação Zurquí, parátipo fêmea, 15.X.1985, M. M. Chavarria col. (INBio); Pérez Zeledón, Estação Santa Elena, 1600 m, parátipo fêmea, 21-29.VII.1999, J. Rodríguez col. (MZSP). PANAMÁ (Fig. 13), Chiriquí: Trilha La Culebra, 1463 m, 849.172’ N $82^{\circ} 27.325^{\prime} \mathrm{W}$, macho, 21.VIII.08, Gillogly \& Smith col. (USNM); idem, macho, 21.VIII.08, Gillogly \& Smith col. (MZSP); Las Nubes, Parque Nacional La Amistad, 8 km NW Cerro Punta, 854’05”N - 82³7’13”'W, 2100 m, macho, 1.VIII.1999, Gillogly col. (MIUP); idem, fêmea, 1.VIII.1999, Gillogly col. (STRI); Reserva La Fortuna, Estação Biológica, 1100 m, atraído por luz, macho, 25-29.VI.1996, Gillogly col. (CASC); idem, fêmea, 12-13. VII.1996, Gillogly col. (TAMU); idem, $8^{\circ} 43.2^{\prime} \mathrm{N}-82^{\circ} 14.247^{\prime} \mathrm{W}, 1225 \mathrm{~m}, 2$ , 29.VIII-02.IX.2008, S. M. Smith col. (ARCO).

\section{Parandra (Parandra) glabra (De Geer, 1774)}

(Figs. 6, 11-13)

Attelabus glaber De Geer, 1774: 352.

Hesperandra (Zikandra) glabra; Monné, 2006: 13 (cat.); Wappes et al., 2006: 4

(distrib.); Santos-Silva, 2007: 8, 9, Figs. 10-13, 15, 17 (chave).

Parandra (Parandra) glabra; Santos-Silva \& Shute, 2009: 31.

Espécie com ampla distribuição e várias formas atreladas à localização geográfica. Os espécimes provenientes do Panamá 

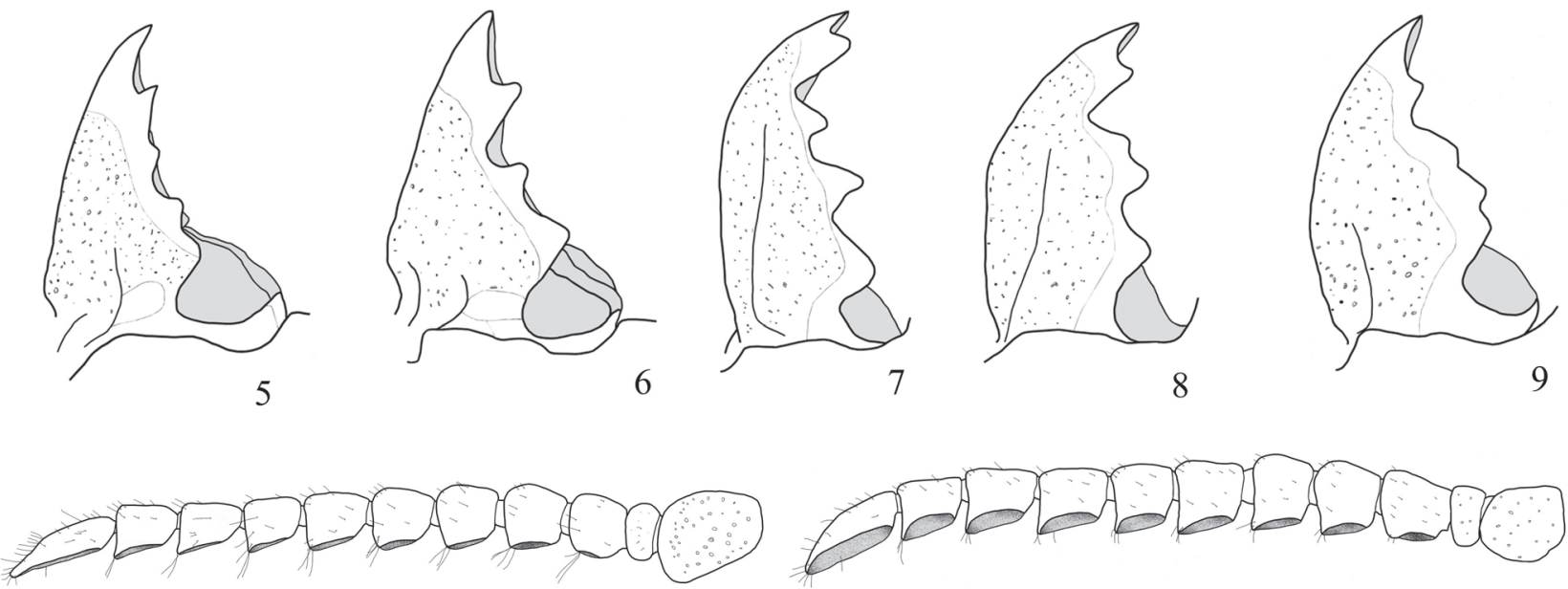

10

11

Figs. 5-11. Mandíbulas: 5, Parandra (Parandra) gilloglyi sp. nov., parátipo fêmea; 6, P. (P.) glabra, fêmea; 7-9, Birandra (Birandra) boucheri sp. nov.: 7, holótipo macho; 8, parátipo macho; 9, parátipo fêmea. Antenas: 10, P. (P.) gilloglyi sp. nov., parátipo macho; 11, P. (P.) glabra, macho.

possuem o submento com pontuação grosseira e abundante, caráter compartilhado com os espécimes encontrados na Costa Rica, Equador e Colômbia. Em contrapartida, nos espécies que ocorrem no Sul e Sudeste do Brasil, a pontuação do submento é nitidamente fina e, não raro, dispersa.

Material examinado. COSTA RICA (Fig. 12), Guanacaste: Estação Mengo, Área de Conservação Guanacaste, Parque Nacional Guanacaste, Vulcão Cação, bosque primário, $1000 \mathrm{~m}$, fêmea, 13.VI.1987, Janzen col. (MZSP). Cartago: Grano de Oro, $1120 \mathrm{~m}$, Chirripo, Turrialba, fêmea, 8-10. VII.1992, P. Campos col. (MZSP). Alajuela: Reserva Biológica Alberto Manuel Brenes, Rio San Lorencito, 850 m, 2 machos, 30.VI-5.VII.1999, J. Rodríguez col. (MZSP). Heredia: Finca la Selva, $3 \mathrm{~km} \mathrm{~S}$ Puerto Viejo, $10^{\circ} 26^{\prime} \mathrm{N}-84^{\circ} 01^{\prime} \mathrm{W}$, macho, 12.IV.1983, H. A. Hespenheide col. (MZSP). Puntarenas: Isla del Coco, Bahia Wafer, Rio Genio, macho, 29.III.1983, T. W. Sherry \& T. K. Werner col. (MZSP). PANAMÁ (Fig. 13), Chiriquí: Rio Colorado, 16,4 km W Volcan, 1125 m, fêmea, VI.1996, A. Rodriguez col. (STRI); Reserva La Fortuna, $8^{\circ} 45.468^{\prime \prime} \mathrm{N}-82^{\circ} 15.671^{\prime} \mathrm{W}$, trilha entre 1234 e 1280 m, 1 macho, 1 fêmea, 1.IX.2008, Gillogly col. (MZSP). Veraguas: $8 \mathrm{~km} \mathrm{~W}$ Santa Fé, Cerro Tute, $950 \mathrm{~m}, 8^{\circ} 30^{\prime} 26^{\prime \prime} \mathrm{N}-81^{\circ} 6^{\prime} 49^{\prime \prime} \mathrm{W}$, macho, 24.VII.1999, Gillogly col. (CASC). Darién: Cana, "Field Station", $7^{\circ} 45.367^{\prime} \mathrm{N}-77^{\circ} 41.050^{\prime} \mathrm{W}, 530 \mathrm{~m}$, macho, 2.V.2008, Gillogly col. (TAMU); idem, macho, 3.V.2008, Gillogly col. (MIUP); idem, fêmea, 3.V.2008, Gillogly col. (USNM); idem, Campo Pirre, $7^{\circ} 45.825^{\prime} \mathrm{N}-77^{\circ} 43.325^{\prime} \mathrm{W}, 1320$ m, 2 machos, 5.V.2008, Gillogly col. (USNM). Bocas del Toro: Miramar, $9^{\circ} \mathrm{N}-82^{\circ} 15^{\prime}$ W, fêmea, VII.1979, H. Wolda col. (ACMT).

Chave para as espécies de Parandra (Parandra) presentes no Panamá

1. Mandíbulas falciformes ou subfalciformes. Machos ...... 2

Mandíbulas não falciformes. Fêmeas 4

2. Antenas (Fig. 10) notavelmente estreitadas para o ápice e não atingem o meio do protórax

$P$. (P.) gilloglyi sp. nov.

Antenas (Fig. 11) não ou fracamente estreitadas para o ápice e ultrapassam o meio do protórax 3

3. Submento com pontuação fina em toda extensão ou moderadamente grossa apenas nas laterais; metepisternos com pelos em toda extensão (esparsos ou muito esparsos a partir do quarto próximo das mesocoxas) ............ P. (P.) solisi (Santos-Silva, 2007)

Submento com pontuação grosseira em toda extensão; metepisternos com pelos apenas no quarto próximo às mesocoxas P. (P.) glabra (De Geer, 1774)

4. Margem interna das mandíbulas sem concavidade acentuada na região mediana (Fig. 5)

$P$. (P.) gilloglyi sp. nov.

Margem interna das mandíbulas com concavidade acentuada na região mediana. (Fig. 6) ...................... 5

5. Dente distal da margem interna das mandíbulas largo e truncado no ápice .... P. (P.) solisi (Santos-Silva, 2007)

Dente distal da margem interna das mandíbulas estreito e aguçado no ápice P. (P.) glabra (De Geer, 1774)

\section{Parandra (Tavandra) polita Say, 1835}

(Figs. 12, 13)

Parandra polita Say, 1835: 192.

Hesperandra (Tavandra) polita; Monné, 2006: 11 (cat.); Wappes et al., 2006: 4 (distrib.).

Parandra (Tavandra) polita; Santos-Silva \& Shute, 2009: 31.

Espécie com ampla área de distribuição, que abrange dos Estados Unidos até a América do Sul (Guiana, Colômbia, Equador, Peru e Bolívia). Reportada pela primeira vez para o Panamá por Bates (1884).

Acreditamos que Parandra (Tavandra) polita é a única espécie do subgênero que ocorre no Panamá. $\mathrm{O}$ registro de Parandra (Tavandra) longicollis Thomson, 1861, para toda a América Central, provavelmente, deve-se a identificação incorreta de espécimes de $P$. (T.) polita e/ou $P$. (T.) brachyderes Lameere, 1902.

Material examinado. COSTA RICA (Fig. 12), Alajuela: Reserva Biológica Alberto Manuel Brenes, San Ramón, 550 m, macho, 26.XI.2003, 
H. J. Lezama col. (MICR); idem, 800 m, macho, 24.IV.2006, H. J. Lezama col. (MICR). Puntarenas: Sendero Bajo Tigre, Monteverde, 1320 m, 2 fêmeas, 4.VIII.1991, H. J. Lezama col. (MICR). PANAMÁ (Fig. 13), Chiriquí: 5 km NE Santa Clara ("Hartmann's"), 850.68'N - 8244.58'W, 1550 m, macho, 24.VIII.2008, Gillogly \& Smith col. (MZSP).

\section{Birandra (Yvesandra) angulicollis (Bates, 1879)}

(Figs. 12-13)

Parandra angulicollis Bates, 1879: 2.

Parandra (Parandra) angulicollis; Monné, 2006: 16 (cat.); Cardona-Duque et al., 2007: 44 (chave).

Birandra (Yvesandra) angulicollis; Santos-Silva \& Shute, 2009: 32.

Originalmente descrita da Guatemala, é encontrada do México (Veracruz) ao Panamá. Foi registrada para esse último país por Bates (1884) e permanece como a única espécie do subgênero encontrada nele.

Material examinado. COSTA RICA(Fig. 12), Puntarenas: Coto Brus, Las Alturas, $8^{\circ} 57^{\prime} \mathrm{N}-82^{\circ} 52^{\prime} \mathrm{W}$, macho, 26-29.III.2002, A. Cline \& A. Tishechkin col. (MZSP); Zona Protetora Arenal-Monteverde, 1500 m, fêmea, 29.V.1979, H. \& A. Howden col. (MZSP); idem, Estación Biológica Monteverde, 1600 m, fêmea, 28.V-14.VI.1999, J. Rodríguez col. (INBio). Heredia: 10 km SE La Virgem, 450-550 m, $10^{\circ} 20^{\prime} \mathrm{N}-84^{\circ} 05^{\prime} \mathrm{W}$, macho, 17.IV.2003, INBioOET-ALAS col. (INBio). Guanacaste: Estación Pitilla, Volcán Cacao, 700 m, macho, 4.IV.1995, H. J. Lezama col. (MZSP). PANAMÁ (Fig. 13), Darién:

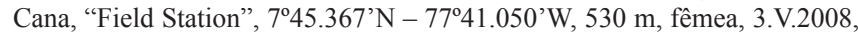
Gillogly col. (MZSP); idem, fêmea, 3.V.2008, Gillogly col. (STRI); idem, macho, 3.V.2008, Gillogly col. (USNM); idem, fêmea, 3.V.2008, Gillogly col. (MIUP); idem, fêmea, 3.V.2008, Gillogly col. (CASC). Coclé: 3 km N El Valle, Cerro Gaital, 8³7’35”N - 806’53”W, 900 m, macho, 21.VII.1999, Gillogly col. (MZSP). Veraguas: $8 \mathrm{~km}$ W Santa Fé, Cerro Tute, $950 \mathrm{~m}$, 830'26"N - 81'6'49”'W, fêmea, 24.VII.1999, Gillogly col. (USNM). Panamá: Cerro Campana, macho, 31.V.1986, B. C. Ratcliffe col. (ACMT); idem, $700 \mathrm{~m}$, ô, V.2002, D. Curoe col. (ACMT). Chiriqui: Lagunas, $5 \mathrm{~km} \mathrm{SW}$ Hato del Volcán, 1360 m, macho, 22.V.1977, S. \& J. Peck. col. (MZSP).

\section{Birandra (Birandra) boucheri sp. nov.}

(Figs. 3-4, 7-9, 12-13)

Etimologia. Espécie dedicada a Stéphane Boucher, coletor de um dos parátipos.

Tegumento castanho-escuro; são enegrecidos: parte frontodorsal da cabeça, genas, mandíbulas, margens do pronoto, margens do escutelo, sutura elitral, margens dos episternos e das cavidades coxais, extremo apical dos fêmures e partes das tíbias.

Macho (Fig. 3). Face dorsal da cabeça com pontos finos, moderadamente abundantes na região central entre o clípeolabro e a borda posterior dos olhos, gradualmente mais grossos em direção aos lobos oculares superiores, área atrás dos olhos e occipício (esparsos em direção a este último); declividade entre a fronte e o clípeo-labro gradual; região central do clípeo-labro projetada para frente, com ápice largo e truncado. Olhos fortemente convexos; borda anterior sem concavidade; borda posterior nitidamente destacada da área lateral atrás dos olhos; lobos oculares superiores fracamente mais estreitos do que os inferiores. Submento glabro, com pontos grossos, abundantes e não confluentes; margem anterior não elevada.

Mandíbulas aproximadamente tão longas quanto à

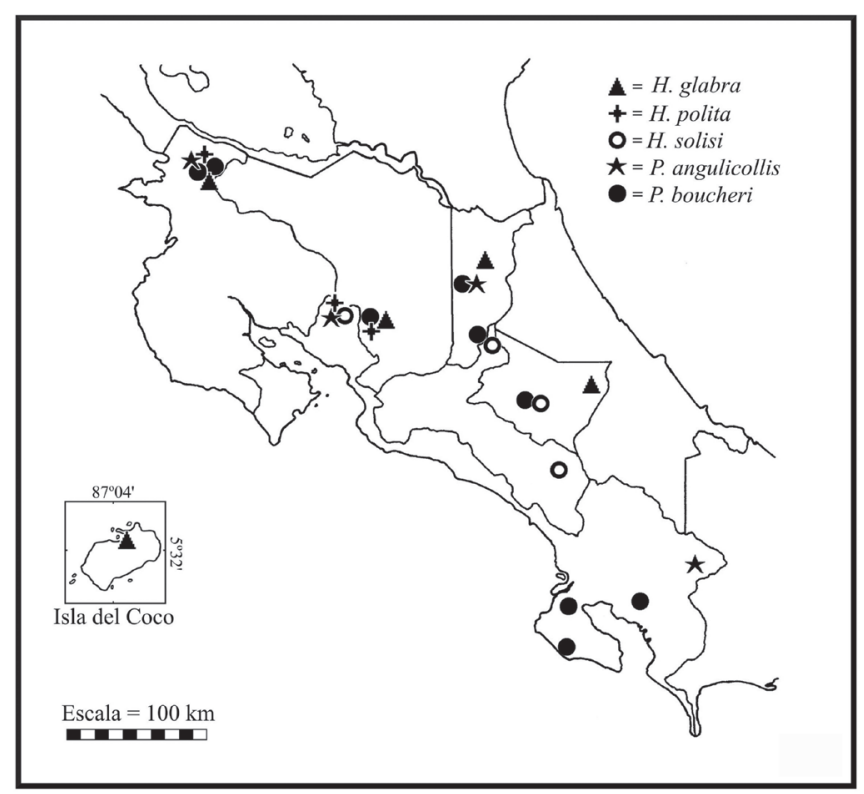

Fig. 12. Mapa de ocorrências de Parandra e Birandra na Costa Rica.

cabeça; margem interna, nos machos major (Fig. 7), com dois dentes grandes, não juntamente protraídos, dos quais, o mais basal é sempre maior; nos machos minor (Fig. 8), os dentes da margem interna são juntamente protraídos e o conjunto é mais largo na mandíbula esquerda do que na direita. Antenas atingem o quarto apical do protórax; antenômero XI aproximadamente tão longo quanto IX-X reunidos; área sensorial dorsal do antenômero XI pequena, elíptica, rasa; pilosidade moderadamente longa e dispersa.

Protórax transversal. Pronoto com pontuação abundante, fina no centro do disco, apenas mais grossa em direção às laterais; ângulos laterais bem marcados, obtusos; ângulos anteriores e posteriores salientes. Élitros com pontuação grossa e abundante, mais fina e mais abundante no terço apical. Metepisternos glabros; pontuação grossa e abundante próximo às cavidades mesocoxais, gradualmente mais dispersa em direção às cavidades metacoxais. Metasterno com pontos grossos nas laterais.

Fêmea (Fig. 4). Região central do clípeo-labro projetada para frente, com ápice estreito e truncado. Submento com pontuação grossa e abundante, principalmente na região central próxima do mento. Mandíbulas (Fig. 9) com os dentes da margem interna juntamente protraídos.

Variabilidade. Tegumento castanho até castanho-escuro. Macho: borda anterior dos olhos fracamente emarginada; submento com pontos confluentes, principalmente em direção ao mento; margem anterior do submento suavemente elevada em machos minor; mandíbulas em machos minor apenas mais curtas do que a cabeça; antenas atingem quase a base do protórax; pontuação dos dois terços basais dos élitros moderadamente esparsa; metepisternos com pontuação grossa em toda extensão; pontuação das laterais do metasterno fracamente grossa. Fêmea: região central do clípeo-labro com ápice de subaguçado até estreitamente truncado. 


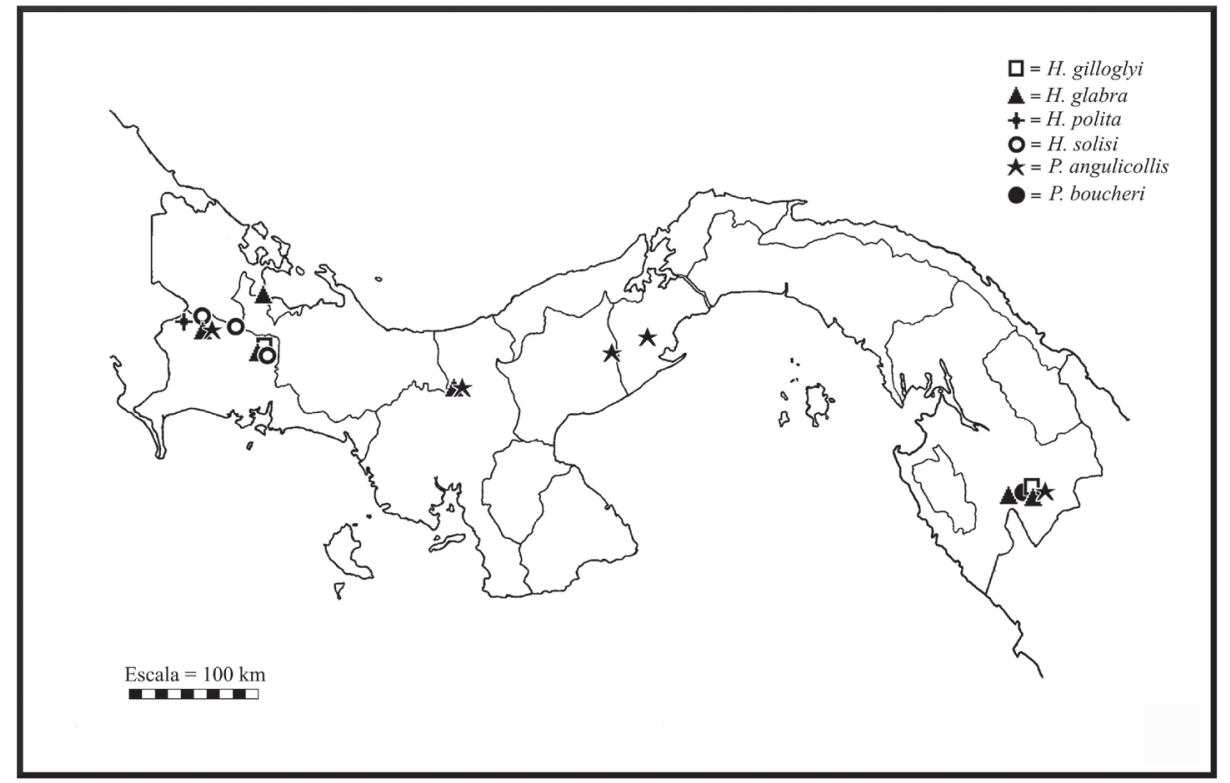

Fig. 13. Mapa de ocorrências de Parandra e Birandra no Panamá.

Dimensões em mm (macho/fêmea). Comprimento total (incluindo mandíbulas), 15,3-21,9/15,8-22,5; largura do protórax entre os ápices dos ângulos anteriores 4,0-6,3/4,15,5; largura umeral, 4,2-5,8/4,5-6,9; comprimento elitral, $9,0-11,8 / 9,5-14,3$.

Material-tipo. Holótipo macho, procedente do PANAMÁ (Fig. 13), Darién: Cana, "Field Station", $7^{\circ} 45.367^{\prime} \mathrm{N}-7^{\circ} 41.050 \mathrm{~W}, 530 \mathrm{~m}, 3 . \mathrm{V} .2008$, Alan R. Gillogly col. (USNM). 29 Parátipos (14 machos, 15 fêmeas) COSTA RICA (Fig. 12), Puntarenas: Golfito, camino para "las Torres", 400500 m, L-S-289300 - 555700, C.B. INB0003836878, macho, 28.IV.2004, B. Gamboa col. (INBio); idem, El Naranjal, $30 \mathrm{~m}$, em tronco podre, macho, [sem data de coleta], S. Boucher col. (MICR); Parque Nacional Corcovado, Estación Sirena, L-S-270500 - 508300, 0-100 m, C.B. CRI000278228, fêmea, VI.1990, N. Obando col. (INBio); idem, C.B. CRI000670469, fêmea, VI.1991, G. Fonseca col. (INBio); idem, C.B. CRI000927244, fêmea, V.1992, G. Fonseca col. (INBio); idem, C.B. CRI000794083, fêmea, IV.1992, G. Fonseca col.(INBio); idem, C.B. CRI000714387, macho, VI.1992, G. Fonseca col. (INBio); idem, C.B. CRI001332578 e CRI001332577, 1 macho, 1 fêmea, III.1993, G. Fonseca col. (INBio); idem, C.B. CRI001843036, fêmea, I.1994, G. Fonseca col. (INBio); idem, Praia Sirena, C.B. INB0003169400, fêmea, 19.II.1999, A. Azofeifa col. (INBio); Rancho Quemado, Peninsula Osa, L-S-292500 - 511000, C.B. CRI000577335, macho, III.1991, F. Quesada col. (INBio); idem, C.B. CRI000577328, fêmea, III.1991, F. Quesada col. (MZSP); Sendero Ajo, Estación Agujas, 300 m, L-S-276750 - 526550, C.B. CRI002412192, macho, 6-12.I.1998, M. Lobo col. (INBio); idem, C.B. CRI002415007 e CRI002415006, 2 machos, 7-12.I.1998, A. Azofeifa col. (INBio); Sendero Zamia, Estación Agujas, 300 m, L-S-279750 - 526550, C.B. CRI002412234, fêmea, 6-12.I.1998, M. Lobo col. (INBio). Guanacaste: Parque Nacional Guanacaste, Estación Pitilla, 9 km S Santa Cecília, 700 m, L-N-220300 - 380200, C.B. CRI000610285, macho, IX.1991, P. Rios col. (MZSP); idem, C.B. CRI000460459, macho, IX.1991, C. Moraga col. (INBio). Cartago: Parque Nacional Tapantí, 1150 m, L-N-194000 - 559800, C.B. CRI001765978, fêmea, VII.1993, G. Mora col. (INBio). Alajuela: Reserva Biológica San Ramón, 800 m, L-N-245100 - 472100, C.B. CRI002461147, fêmea, III.1997, G. Carballo col. (INBio); Reserva Biológica Alberto Manuel Brenes, Rio San Lorencito, 850 m, L-N-245500 - 470800, C.B. INB0003034963, fêmea, 19-26.III.1999, A. Azofeifa col. (INBio); idem, 800 m, fêmea, 23-25.IV.2003, H. J. Lezama col. (MICR). Heredia: Parque Nacional Braulio Carrillo, 400-600 m, L-S-527700 - 256500, C.B. CRI000176266, fêmea, III.1990, C. Chaves col. (INBio); 15 km SSE La Virgem, $950-1050 \mathrm{~m}, 10^{\circ} 17^{\prime} \mathrm{N}-84^{\circ} 05^{\prime} \mathrm{W}$, C.B. INB0003665510, macho,
18.IV.2001, INBio-OET-ALAS col. (INBio). PANAMÁ (Fig. 13), Darién:

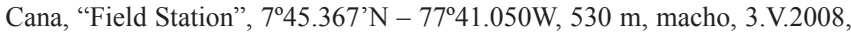
Alan R. Gillogly col. (MZSP); idem, fêmea, 3.V.2008 (MIUP); idem, macho, 8.V.2008, Alan R. Gillogly col. (MZSP); idem, macho, 8.V.2008, Alan R. Gillogly col. (CASC). C.B. = Código de barras.

Discussão. Além de B. (Birandra) boucheri sp. nov., apenas mais uma espécie desse subgênero, caracterizado pela ausência de carena na área sensorial das antenas, é conhecida da América Central: B. (Birandra) mariahelenae (SantosSilva, 2002), descrita da Jamaica. As duas espécies podem ser reconhecidas pelos caracteres apresentados na chave abaixo. A nova espécie difere de B. (Birandra) punctata (White, 1853): protórax mais largo nos dois sexos (nos machos, largura igual a 1,6 ou 1,7 vezes o comprimento; nas fêmeas, igual a 1,5 vezes o comprimento), com as margens laterais não ou fracamente curvadas para dentro no terço próximo à cabeça; mandíbula nos machos major com dois dentes grandes, separados na margem interna e juntamente protraídos nos machos minor (dente mais basal maior do que o mais distal). Em B. (Birandra) punctata, o protórax é mais estreito nos dois sexos (nos machos, largura igual a 1,5 vezes o comprimento; nas fêmeas, igual a 1,4 vezes o comprimento), com as margens laterais do terço próximo à cabeça nitidamente curvadas para dentro, os machos major apresentam apenas um dente pequeno na margem interna das mandíbulas e, nos machos minor, os dentes da margem interna não são juntamente protraídos e o dente mais basal é menor do que o mais distal.

Chave para as espécies de Birandra (Birandra) da América Central

1. Margem anterior dos olhos com concavidade nítida nos dois sexos; margem interna da mandíbula dos machos sem dentes. Jamaica Birandra

(Birandra) mariahelenae (Santos-Silva, 2002) 
Margem anterior dos olhos sem concavidade nos dois sexos; margem interna da mandíbula dos machos com dentes. Costa Rica e Panamá

$$
\text { Birandra (Birandra) boucheri sp. nov. }
$$

Agradecimentos. A Alan R. Gillogly (OJSM) pela oportunidade de estudar o material e pela doação de todos os espécimes para diversos museus. A Aaron Smith e Gary L. Parsons (ARCO), Angel Solis (INBio) e James Wappes (ACMT) pelo empréstimo de material adicional para estudo.

\section{REFERÊNCIAS}

Bates, W. H. 1879. Biologia Centrali-Americana, Insecta, Coleoptera. London, 5: 1-16.

Bates, W. H. 1884. Biologia Centrali-Americana, Insecta, Coleoptera. Supplement to Longicornia. London, 5: 225-248.

Cardona-Duque, J.; A. Santos-Silva \& M. Wolff. 2007. A new species of Parandrinae from the Central Andes of Colombia (Coleoptera: Cerambycidae). Zootaxa 1661: 39-45.

Chemsak, J. A. 1996. Illustrated Revision of the Cerambycidae of North America. Volume I. Parandrinae, Spondylidinae, Aseminae, Prioninae. Burbank, Wolfsgarden Books, 1: i-x +150 p.
De Geer, C. 1774. Mémoires pour servir à l'histoire des insectes. Stockholm, Imp. Pierre Hesselberg, 4, xii +456 p.

Monné, M. A. 2002. Catalogue of the Neotropical Cerambycidae (Coleoptera) with known host plant - part V: subfamilies Prioninae, Parandrinae, Oxypeltinae, Anoplodermatinae, Aseminae and Lepturinae. Publicações Avulsas do Museu Nacional 96: 1-70.

Monné, M. A. 2006. Catalogue of the Cerambycidae (Coleoptera) of the Neotropical Region. Part III. Subfamilies Parandrinae, Prioninae, Anoplodermatinae, Aseminae, Spondylidinae, Lepturinae, Oxypeltinae, and addenda to the Cerambycinae and Lamiinae. Zootaxa 1212: $1-244$.

Monné, M. A. \& E. F. Giesbert. 1994. Checklist of the Cerambycidae and Disteniidae (Coleoptera) of the Western Hemisphere. California, Wolfsgarden Books. Burbank, xiv +410 p.

Santos-Silva, A. 2007. Nova espécie de Hesperandra (Zikandra) Santos-Silva e chave para o subgênero (Coleoptera, Cerambycidae, Parandrinae). Les Cahiers Magellanes 66: 1-11.

Santos-Silva, A. \& S. Shute. 2009. The identity of Parandra laevis Latreille, 1804 and nomenclatural changes in the Parandrinae (Coleoptera: Cerambycidae). Zookeys 25: 19-35.

Say, T. 1835. Descriptions of new North American coleopterous insects, and observations on some already described. Boston Journal of Natural History 1: 151-203.

Wappes, J. E.; R. F. Morris; E. H. Nearns \& M. C. Thomas. 2006. Preliminary checklist of Bolivian Cerambycidae (Coleoptera). Insecta Mundi 20: $1-45$. 